\title{
Reducing Cardiovascular Morbidity and Mortality With the Statins
}

\author{
Rex W. Force, PharmD
}

Editors' Note: This month we introduce a new feature to The Journal-STEPped Care: An Evidence-Based Approach to Drug Therapy. These articles are designed to provide concise answers to the drug therapy questions that family physicians encounter in their daily practice. The format of the feature will follow the mnemonic STEP: safety (an analysis of adverse effects that patients and providers care about), tolerability (pooled drop-out rates from large clinical trials), effectiveness (bow well the drugs work and in what patient population[s]), and price (costs of drug, but also cost effectiveness of therapy). ${ }^{1}$ Hence, the name STEPped Care.

Since the informatics pioneers at McMaster University introduced evidence-based medicine, ${ }^{2}$ Slawson and Shaughnessy $y^{3,4}$ bave brought it to mainstream family medicine education and practice. This feature is designed to further the mission of searching for the truth in medical practice. Authors will provide information in a structured format that allows the readers to get to the meat of a therapeutic issue in a way that can belp physicians (and patients) make informed decisions. The articles will discourage the use of disease-oriented evidence (DOE) to make treatment decisions. Examples of DOEs include blood pressure lowering, decreases in bemoglobin $A_{l c}$, and so on. We will include studies that provide POEMspatient-oriented evidence that matters (myocardial infarctions, pain, strokes, mortality, etc)-with the goal of offering patients the most practical, appropriate, and scientifically substantiated therapies. Number needed to treat to observe benefit in a single patient will also be included as a way of defining advantages in terms that are relatively easy to understand. 5,6

At times this effort will be frustrating. Even as vast as the

The hydroxymethylglutaryl-coenzyme A (HMG$\mathrm{CoA})$ reductase inhibitors are recommended as major drugs in the treatment of hyperlipidemia by the second National Cholesterol Education Program (NCEP II) Expert Panel. ${ }^{1}$ These agents lower cholesterol by inhibiting the rate-limiting enzyme (HMG-CoA reductase) responsible for

Submitted, revised, 3 September 1997.

From the Department of Family Medicine, Idaho State University, Pocatello. Address reprint requests to Rex W. Force, PharmD, Department of Family Medicine, Idaho State University, Campus Box 8357, Pocatello, ID 83209-8357. biomedical literature is, it does not always support what clinicians do. We will avoid making conchusions that are not supported by POEMs. Nevertheless, POEMs should be incorporated into clinical practice. The rest is up to the reader. Blending POEMs with rational thought, clinical experience, and importantly, patient preferences can be the essence of the art of medicine.

We bope you will find these articles useful and easy to read. Your comments and suggestions are welcome. You may contact the editors through the editorial office of $\mathcal{7} A B F P$ or on the Internet (bttp://clinic.isu.edu/drugsteps/intro.html). We bope the articles provide you with useful information that can be applied in everyday practice, and we look forward to your feedback.

Rex W. Force, PharmD, STEPped Care Feature Editor John Geyman, MD, Editor Fournal of the American Board of Family Practice

\section{References}

1. Shaughnessy AF, Slawson DC, Bennett JH. Separating the wheat from the chaff: identifying fallacies in pharmaceurical promotion.J Gen Intern Med 1994;9:563-8.

2. Evidence-based medicine: a new approach to teaching the practice of medicine. Evidence-Based Medicine Working Group. JAMA 1992;268:2420-5.

3. Slawson DC, Shaughnessy AF, Bennett JH. Becoming a medical information master: feeling good about not knowing everything. J Fam Pract 1994;38:505-13.

4. Shaughnessy AF, Slawson DC, Bennett JH. Becoming an information master: a guidebook to the medical information jungle. J Fam Pract 1994;39:489-99.

5. Laupacis A, Sackett DL, Roberts RS. An assessment of clinically useful measures of the consequences of treatment. $N$ Engl J Med 1988;318:1728-33.

6. Wiffen PJ, Moore RA. Demonstrating effectiveness-the concept of numbers-needed-to-treat. J Clin Pharm Ther $1996 ; 21: 23-7$. cholesterol synthesis in the liver. Secondarily, a compensatory mechanism of increasing low-density lipoprotein (LDL) receptors acts to decrease circulating LDL concentrations. ${ }^{2}$ Five HMGCoA reductase inhibitors, or so-called "statins," are currently on the market: lovastatin (Mevacor), pravastatin (Pravachol), simvastatin (Zocor), fluvastatin (Lescol), and atorvastatin (Lipitor). These drugs differ in their pharmacokinetic profiles, potencies, and costs.

For several years controversy existed as to whether cholesterol lowering with pharmacotherapy was beneficial to patients. ${ }^{3,4}$ A great deal 
of information was available regarding the efficacy of the statins in lowering total cholesterol and LDL while raising high-density lipoprotein (HDL). This information, however, was diseaseoriented evidence, meaning that data were not available regarding more important outcomes (decreased myocardial infarctions (MIs), strokes, and mortality). More recently, three trials ${ }^{5-7}$ with the statins have provided evidence that some agents might be useful for primary and secondary prevention of cardiovascular events and mortality. Importantly, these studies have provided the patient-oriented evidence that matters (POEMs) to patients and providers.

\section{Methods}

A MEDLINE search was performed for January 1983 through June 1997 using the search terms "anticholesteremic agents," "HMG-CoA reductase inhibitors," "cardiovascular diseases," "myocardial infarction," "mortality," and "survival." The search was limited to human clinical trials with the statins published in English language journals. Studies were selected if they had POEMs (cardiovascular disease, mortality, coronary artery bypass grafts [CABG], etc) as primary outcomes. The number needed to treat to prevent one negative outcome was then calculated and presented in tabular form. For completeness, other references reviewing the pharmacology and use of the statins were included at the discretion of the author. This review will examine the statins and their role in lowering plasma lipid levels to prevent cardiovascular disease by using the STEP approach: safety (an analysis of adverse effects that patients and providers care about), tolerability (pooled dropout rates from large clinical trials), effectiveness (how well the drugs work and in what patient population[s]), and price (costs of drug, but also cost effectiveness of therapy).

\section{Safety and Tolerability}

The safety of the statins has been well-established and has been recently reviewed elsewhere. ${ }^{2}$ More than 8000 patients received lovastatin for 48 weeks in the Expanded Clinical Evaluation of Lovastatin (EXCEL) trial. 8 ,9 The most common adverse effect in EXCEL was constipation. Nevertheless, the incidence of constipation in the lovastatin-treated patients ( 6.0 percent) was only slightly higher than placebo (4.7 percent). Infre- quent elevations of serum aminotransferases (alanine aminotransferase and aspartate aminotransferase) and creatine kinase did occur in the lovastatin group. These elevations were more common at doses of 40 to $80 \mathrm{mg} / \mathrm{d}$. From these data about 2 to 6 percent of patients receiving more than $40 \mathrm{mg} / \mathrm{d}$ would have an elevation (two times normal) in aminotransferases and approximately 0.2 to 0.5 percent would have an elevation (10 times normal) in creatine kinase. Actual hepatitis or rhabdomyolysis are extremely uncommon; therefore, these increased laboratory values appear to have limited clinical significance. About 5.1 percent of the patients on placebo dropped out of the trial because of adverse events whereas 6.3 to 7.0 percent of patients receiving lovastatin regimens discontinued treatment because of adverse effects. Overall, about 85 percent of patients receiving placebo and lovastatin finished the 48week trial.

Subsequent studies with simvastatin ${ }^{5}$ and pravastatin $^{6,7}$ have shown that these agents are welltolerated. In the Scandinavian Simvastatin Survival Study (4S), ${ }^{5} 6$ percent of patients in both the placebo and simvastatin $(40 \mathrm{mg} / \mathrm{d})$ groups discontinued therapy because of adverse events during the 5-year trial. Specifically, serum aminotransferase elevations were not more common in the simvastatin group, and no cases of hepatitis were reported. Creatine kinase elevations occurred in 6 of 2221 ( 0.3 percent) and in 1 of $2223(0.05$ percent) of patients receiving simvastatin or placebo, respectively. A single patient developed rhabdomyolysis in the simvastatin group. Additionally, there were no differences between the groups with regard to cancers. Overall, 13 percent of patients stopped taking their tablets in the placebo group compared with 10 percent of patients receiving simvastatin.

In the West of Scotland Coronary Prevention Study (WOSCOPS) ${ }^{6}$ with pravastatin $(40 \mathrm{mg} / \mathrm{d})$, there were no significant differences in serum aminotransferase elevations, creatine kinase elevations, cancer development, or myalgias in the patients receiving pravastatin compared with those receiving placebo. Overall, 30.8 percent and 29.6 percent of patients withdrew from the 5-year study in the placebo and pravastatin groups, respectively. In a second pravastatin study, the Cholesterol and Recurrent Events (CARE) Trial, ${ }^{7} 86$ percent and 94 percent of the patients in the 
Table 1. Number Needed to Treat to Prevent a Single Adverse Outcome for Primary Prevention (WOSCOPS) and Secondary Prevention (CARE and 4S) Trials with the Statins Compared with Placebo.

\begin{tabular}{lccc}
\hline Events & WOSCOPS & Number Needed to Treat & CARE \\
\hline Coronary artery bypass graft, angioplasty & 125 & 21 & 17 \\
Acute myocardial infarction & $42^{*}$ & $33^{*}$ & 21 \\
Stroke & $\mathrm{NS}$ & 83 & 63 \\
Death from coronary heart disease & $\mathrm{NS}$ & $\mathrm{NS}$ & 29 \\
Total mortality & $111 \dagger$ & $\mathrm{NS}$ & 30 \\
\hline
\end{tabular}

*Includes nonfatal myocardial infarction and death from coronary heart disease.

$\dagger \mathrm{P}=\mathbf{0 . 0 5 1}$.

WOSCOPS - West of Scotland Coronary Prevention Study, CARE - Cholesterol and Recurrent Events, 4 S - Scandinavian Simvastatin Survival Study.

NS - not significantly different from placebo.

placebo and pravastatin groups, respectively, completed at least 4 years on the study protocol. There were no significant differences in the incidence of serum aminotransferase elevation, creatine kinase elevation, or myositis. The placebo group developed 161 cancers, whereas the pravastatin group developed $172(P=\mathrm{NS})$. The pravastatin group, however, developed significantly more breast cancers (12 versus $1, P=0.002$ ). The authors speculate that this difference could be due to an unusually low incidence of breast cancer in the control group rather than an increased incidence in the pravastatin group. ${ }^{7}$

Information from smaller trials indicates that fluvastatin and atorvastatin share the risk of elevating serum transaminase and creatine kinase levels, as discussed above for the other statins., ${ }^{2,10}$ At high doses all of these drugs can induce tumors in rats and mice. At present, the clinical importance of this potential in humans is unknown.

Most pharmacokinetic drug interactions with the statins are relatively unimportant clinically. Rhabdomyolysis has occurred in patients receiving the statins in conjunction with gemfibrozil, niacin, cyclosporine, and erythromycin. ${ }^{2}$ The manufacturers of the statins state that patients should be monitored for hepatotoxicity, elevated creatine kinase levels, and muscle weakness.

\section{Effectiveness}

The statins markedly lower total and LDL cholesterol levels while causing mild elevations of HDL cholesterol levels. The potencies of the agents in lowering LDL cholesterol levels differ; maximal recommended doses of lovastatin $(80 \mathrm{mg} / \mathrm{d})$, pravastatin $(40 \mathrm{mg} / \mathrm{d})$, and simvastatin $(40 \mathrm{mg} / \mathrm{d})$ generally decrease LDL cholesterol levels by 25 to
40 percent. Fluvastatin is considered less potent and reduces LDL cholesterol levels by 20 to 25 percent at $40 \mathrm{mg} / \mathrm{d} .^{2}$ The new agent atorvastatin is the most potent statin available and lowers LDL cholesterol by 50 to 60 percent at a dose of 80 $\mathrm{mg} / \mathrm{d}$. Additionally, atorvastatin has the greatest effect on triglycerides when compared with the other statins. ${ }^{10}$ These findings, however, are disease-oriented evidence, and there are no data on the effects of fluvastatin and atorvastatin on cardiovascular morbidity and mortality. Limited data are available on the effect of lovastatin on these patient-oriented outcomes. The statins have not been compared head-to-head to evaluate patientoriented outcomes; the only comparative studies available evaluate lipid-lowering effects. ${ }^{2}$ Until head-to-head morbidity and mortality trials of the statins are completed, clinicians should base their selection of a statin on the available POEMs.

The EXCEL trial was designed to evaluate the cholesterol-lowering efficacy of three different lovastatin regimens or placebo during a 48-week period in 8245 patients. ${ }^{8,9}$ Although the primary goals of the study were to evaluate the efficacy of the drug in lowering lipid levels and to determine the long-term safety of the agent, there were several cardiovascular events and deaths that warrant comment. Of the 36 deaths that occurred, 31 were from coronary heart disease, and 28 of these occurred in the lovastatin groups. ${ }^{3,8,9}$ It should be noted that EXCEL patients were those receiving treatment for primary and secondary prevention. A recent secondary prevention trial of lovastatin, 40 to $80 \mathrm{mg} / \mathrm{d}$ versus $2.5 \mathrm{mg} / \mathrm{d}$, showed that the higher doses were effective in reducing the incidence of CABG. ${ }^{11}$ There was no placebo arm in the lipid-lowering portion of this trial, however. 
Table 2. Cost of Monthly Supply of Statins.

\begin{tabular}{lcc}
\hline Drug & Dose $^{*}(\mathrm{mg})$ & Cost per Month (\$) \\
\hline Lovastatin & 20 & 67.50 \\
Pravastatin & 20 & 58.97 \\
Simvastatin & 10 & 60.86 \\
Fluvastatin & 20 & 36.60 \\
Atorvastatin & 10 & 54.72 \\
\hline
\end{tabular}

*Usual initial dose per day.

From Med Lett Drugs Ther. ${ }^{10}$

Overall, neither mortality nor the occurrence of MIs or cancers was different between the groups. Eighty-five percent of patients complied with the treatment regimen during the 4-year study. ${ }^{11}$

Since 1994 reports of three trials ${ }^{5-7}$ have been published describing POEMs with cholesterol lowering using pravastatin and simvastatin. The effects of these drugs on CABG and angioplasty, acute MI, stroke, death from coronary heart disease (CHD), and overall mortality in patients receiving primary and secondary prevention are summarized in Table 1.

WOSCOPS $^{6}$ was designed as a primary prevention trial in high-risk patients. The trial was conducted in Scotland and consisted of 6545 men (mean age 55 years) who had mean baseline plasma cholesterol and LDL concentrations of $272 \mathrm{mg} / \mathrm{dL}$ and $192 \mathrm{mg} / \mathrm{dL}$, respectively. The patients were randomly assigned to receive $40 \mathrm{mg}$ of pravastatin or placebo each evening in a doubleblind trial design. None of the patients had experienced an MI, and only 5 percent had angina. Forty-four percent were current smokers (an additional 34 percent were ex-smokers), they drank the equivalent of nearly 2 six-packs of beer per week, and 15 percent had hypertension. Their mean HDL level was $44 \mathrm{mg} / \mathrm{dL}$. The primary end point of the study was the combined incidence of nonfatal MIs or death from CHD.

During the 5-year study, 7.9 percent of placebo and 5.5 percent of pravastatin patients experienced a definite primary outcome $(P<0.001)$. The number needed to treat to prevent a single adverse outcome has been calculated in Table 1. For example, to prevent the occurrence of a single definite MI or death from CHD, 42 patients would have to be treated for 5 years with pravastatin 40 $\mathrm{mg} / \mathrm{d}$. Overall mortality was reduced from 4.1 percent in the placebo group to 3.2 percent in the pravastatin group $(P=0.051)$ yielding a number needed to treat of 111.6
Two secondary prevention trials also showed the benefit of cholesterol-lowering therapy with statins. The CARE trial ${ }^{7}$ was a multicenter, dou- $\frac{0}{2}$ ble-blind trial investigating the effects of pravastatin on coronary events after MI. Pravastatin (40 $\mathrm{mg} / \mathrm{d}$ ) or placebo was administered to 3583 men and 576 women who had mean baseline total cholesterol and LDL concentrations of $209 \mathrm{mg} / \mathrm{dL}$ and $139 \mathrm{mg} / \mathrm{dL}$, respectively. Their mean age was 59 years and mean HDL concentration was 39 $\mathrm{mg} / \mathrm{dL}$. Fifteen percent had diabetes, 43 percent had hypertension, and 21 percent were current smokers. The patients were a mean of 10 months post-MI. Like WOSCOPS, the primary end point of the study was the combined incidence of nonfatal MI or death from CHD. During the 5-year study period, 13.2 percent of placebo and 10.2 percent of pravastatin patients experienced a primary outcome $(P=0.003)$, yielding a number needed to treat of 33 for 5 years. Overall mortality was not significantly reduced in the pravastatin group. Interestingly, patients who had baseline LDL concentrations of less than $125 \mathrm{mg} / \mathrm{dL}$ did not experience any benefit from pravastatin therapy. This finding calls into question the use of $\vec{\bullet}$ statins to reduce cholesterol to less than $100 œ$ $\mathrm{mg} / \mathrm{dL}$ in patients whose cholesterol concentrations are less than $125 \mathrm{mg} / \mathrm{dL}^{7,12}$

The second trial ${ }^{5}$ demonstrating benefit of cholesterol-lowering therapy as secondary prevention, $4 \mathrm{~S}$, was a multicenter, double-blind trial of simvastatin (20 to $40 \mathrm{mg} / \mathrm{d}$ ) in patients with previous MI or angina. At baseline, mean total cholesterol and LDL concentrations were 261 $\mathrm{mg} / \mathrm{dL}$ and $188 \mathrm{mg} / \mathrm{dL}$, respectively. One half of the patients were ex-smokers and about a quarter were current smokers, 26 percent were hypertensive, and 5 percent were diabetic. Two thirds of the patients were within 5 years of their first diag- 은 nosis of angina or MI. The mean age was 59 years $\stackrel{N}{\sigma}$ and mean HDL concentration was $46 \mathrm{mg} / \mathrm{dL}$. ? Eight-hundred twenty-seven women and 3617 men received active drug or placebo and were ob- స్心 served for 5 years with the goal of reducing their total cholesterol concentrations to 116 to $201 \stackrel{\circ}{C}$ $\mathrm{mg} / \mathrm{dL}$.

The primary end point of the study was overall mortality. During the study period, 11.5 percent $\frac{0}{\Phi}$ of patients receiving placebo and 8.2 percent of $\frac{}{\mathbb{Q}}$ patients receiving simvastatin died $(P=0.0003)$, yielding a number needed to treat of 30 . Definite 
Table 3. Drug STEPS Overview.

\begin{tabular}{ll}
\hline $\begin{array}{l}\text { Safety and } \\
\text { Tolerability }\end{array}$ & $\begin{array}{l}\text { Statins are well tolerated and safe. In large clinical trials, pooled drop-out rate is about } 15 \%, \\
\text { not different from placebo. } \\
\text { Effectiveness }\end{array}$ \\
$\begin{array}{l}\text { Simvastatin (in secondary prevention) and pravastatin (in primary and secondary prevention) } \\
\text { have been shown to lower cardiovascular mortality and morbidity in well-defined patient populations. } \\
\text { Price }\end{array}$ & $\begin{array}{l}\text { Statins typically cost } \$ 2 \text { to } \$ 3 \text { per day. Cost effectiveness models are positive. } \\
\text { With appropriate selection, cholesterol lowering with pravastatin and simvastatin can benefit some } \\
\text { patients. }\end{array}$ \\
\hline
\end{tabular}

acute MIs were significantly reduced in the simvastatin group (7.4 versus 12.1 percent with placebo). Based on these data, 21 patients would need to be treated with simvastatin for 5 years to prevent one MI. Other endpoints are provided in Table 1.

An assessment of the initial level of risk to the patient is essential for appropriate use of the statins. 3,4,12 For example, in the CARE trial patients with LDL concentrations less than 125 $\mathrm{mg} / \mathrm{dL}$ did not receive any benefit from pravastatin, while patients with LDL concentrations greater than $150 \mathrm{mg} / \mathrm{dL}$ had a 10 percent absolute reduction in risk of coronary events. In 4S, 59year-old male patients with $\mathrm{CHD}$ and a total cholesterol of $261 \mathrm{mg} / \mathrm{dL}$ would be expected to live 15.6 years without treatment and 15.9 years with 5 years of treatment. ${ }^{13}$ Also of note, most trials of cholesterol lowering required 2 years of continuous medication before the treatment effect emerged. In patients with a life expectancy of less than 2 years, these agents are unlikely to provide a survival benefit.

\section{Price}

The cost of 1 month's supply of the statins is shown in Table 2. Typically these agents cost $\$ 40$ to $\$ 110$ per month depending on the dosing regimen. ${ }^{10}$ Numerous cost-effectiveness analyses have been published within the past 10 years; however, most of them were based on models of cholesterol lowering and the expected benefits on cardiovascular morbidity and mortality. Few data on the cost effectiveness of the statins are available since the above POEM data have been published. Recently Johannesson et a ${ }^{13}$ produced a sophisticated cost-effectiveness analysis based on the $4 \mathrm{~S}$ trial. Depending on patient age and serum cholesterol concentration, simvastatin treatment resulted in varying cost savings per life-year gained for patients with CHD in this model. For example, in younger patients the costs of the interven- tion (simvastatin therapy and monitoring) were significantly less than the direct and indirect costs of the morbidity of coronary disease. In older patients the cost per life-year gained ranged from $\$ 1,200$ to $\$ 13,300$, with men having a lower cost per life-year gained than women. It should be noted that the costs of drugs and of various events (MI, CABG, etc) in Sweden were less than typical costs in the United States.

\section{Summary}

Based on the available literature, pravastatin and simvastatin have a role in lowering total cholesterol and LDL concentrations in patients with existing cardiovascular disease (Table 3). The result of lowering serum lipid levels with these agents is fewer MIs and CABGs. Pravastatin did not lower overall mortality as secondary prevention in patients with average serum cholesterol concentrations, whereas simvastatin did decrease mortality in higher risk patients. Additionally, in very high risk male patients, pravastatin has been shown to be effective for primary prevention of cardiovascular morbidity and mortality. Studies with lovastatin have not shown a benefit in decreasing the incidence of $\mathrm{MI}$ or death. Although one trial did show a decrease in CABGs with secondary preventive therapy with lovastatin, this trial was not placebo-controlled. Data are not available regarding the ability of fluvastatin or atorvastatin therapy to provide POEMs with cardiovascular disease. In general, the drugs are well-tolerated and cost from $\$ 2$ to $\$ 3$ a day.

With appropriate patient selection clinicians can expect simvastatin to provide benefits to patients with existing CHD and pravastatin to provide similar benefits in patients requiring primary or secondary prevention.

\section{References}

1. Summary of the second report of the National Cholesterol Education Program (NCEP) Expert Panel 
on Detection, Evaluation, and Treatment of High Blood Cholesterol in Adults (Adult Treatment Panel II). JAMA 1993;269:3015-23.

2. Hsu I, Spinler SA, Johnson NE. Comparative evaluation of the safety and efficacy of HMG-CoA reductase inhibitor monotherapy in the treatment of primary hypercholesterolemia. Ann Pharmacother 1995;29:743-59.

3. Silberberg JS. After the meta-analyses: a commentary on treatment of dyslipidaemia in the primary prevention of coronary heart disease. Aust NZ J Med 1994;24:717-21.

4. Davey-Smith G, Song F, Sheldon TA. Cholesterol lowering and mortality: the importance of considering initial level of risk. BMJ 1993;306:1367-73.

5. Randomised trial of cholesterol lowering in $444 \mathrm{pa}$ tients with coronary heart disease: the Scandinavian Simvastatin Survival Study (4S). Lancet 1994;344: 1383-9.

6. Shepherd J, Cobbe SM, Ford I, Isles CG, Lorimer AR, MacFarlane PW, et al. Prevention of coronary heart disease with pravastatin in men with hypercholesterolemia. West of Scotland Coronary Prevention Study Group. N Engl J Med 1995;333:1301-7.

7. Sacks FM, Pfeffer MA, Moye LA, Rouleau JL, Rutherford JD, Cole TG, et al. The effect of pravastatin on coronary events after myocardial infarction in patients with average cholesterol levels. Cholesterol and Recurrent Events Trial Investigators.
N Engl J Med 1996:335:1001-9.

8. Bradford RH, Shear CL, Chremos AN, Dujovne C, Downton M, Franklin FA, et al. Expanded Clinical Evaluation of Lovastatin (EXCEL) study results. I. Efficacy in modifying plasma lipoproteins and adverse event profile in 8245 patients with moderate hypercholesterolemia. Arch Intern Med 1991;151:43-9.

9. Bradford RH, Shear CL, Chremos AN, Dujovne CA, Franklin FA, Grillo RB, et al. Expanded Clinical Evaluation of Lovastatin (EXCEL) study results: two-year efficacy and safety follow-up. Am J Cardiol 1994;74:667-73.

10. Atorvastatin-a new lipid-lowering drug. Med Lett Drugs Ther 1997;39:29-31.

11. The effect of aggressive lowering of low-density lipoprotein cholesterol levels and low-dose anticoagulation on obstructive changes in saphenous-vein coronary-artery bypass grafts. The Post Coronary Artery Bypass Graft Trial Investigators. N Engl J Med 1997;336:153-62.

12. Lawless C, Force R. Lipid lowering in post-MI patients with normal cholesterol. J Fam Pract 1997; 44:30.

13. Johannesson $M$, Jonsson B, Kjekshus J, Olsson AG, Pedersen TR, Wedel H. Cost effectiveness of simvastatin treatment to lower cholesterol levels in patients with coronary heart disease. Scandinavian Simvastatin Survival Study Group. N Engl J Med 1997;336:332-6. 